\title{
Ingeniería Tisular Para Regeneración Nerviosa: Una Revisión
}

\section{Tissue Engineering For Nervous Regeneration: A Review}

\author{
Verónica Guardo-Gómez ${ }^{1}$, María Alexandra Fajardo-Perdomo ${ }^{1}$ Ana L. Muñoz ${ }^{2}$, Lina A. \\ Gómez ${ }^{3}$
}

Introduction: This article presents advances in regenerative medicine aimed at the regeneration of nervous and neuronal tissue, focusing on regeneration of neurons, axons and nerve regeneration. We will review the techniques that currently exist, the most used or promising, in the search of advances to regenerate this type of tissues. Objective: With this review we want to describe the current knowledge about regenerative medicine and tissue engineering oriented to nerve tissue repair. Methodology: To carry out this review, a search of articles was carried out between 2007 and 2018, the search was restricted to the articles that they included within their keywords; Tissue Engineering, Neurodegenerative Diseases, Regenerative Medicine, Axonal Regeneration, Neuronal Regeneration, Tissue Regeneration. We will mention about techniques such as implantation.Conclusions: with this review we could observe that most of the mentioned techniques work better when combined, taking advantage of each one to promote a greater regeneration of the different tissues.

Key words: Tissue Engineering, Neurodegenerative Diseases, Regenerative Medicine Rev Chil Neuro-Psiquiat 2020; 58 (1): 50-60

\section{Introducción}

Anev niv nivel mundial, la carga de trastornos neurológicos se ha incrementado en los últimos 25 años debido al envejecimiento de la población, a pesar de la disminución en las tasas de mortalidad por accidentes cerebrovasculares y trastornos neurológicos

Aceptado: 19/03/2020

Recibido: 13/12/2018

Los Autores declaran no tener ningún conflicto de intereses.

1. Estudiantes de Ingeniería Biomédica. Universidad Antonio Nariño. Bogotá. Colombia.

2. Grupo de Biología Celular y Funcional e Ingeniería de Biomoléculas. Facultad de Ciencias.

Universidad Antonio Nariño. Bogotá. Colombia.

3. Centro de Investigación Biomédica (Cibus). Facultad de Medicina. Universidad de la Sabana. Chía. Cundinamarca. Colombia 
transmisibles. ${ }^{1}$

En el sistema nervioso central (SNC) de mamíferos adultos, la mayoría de los axones lesionados no se regeneran, mientras que a nivel del sistema nervioso periférico (SNP) la regeneración de axones a larga distancia y la recuperación funcional sustancial pueden ocurrir. Tanto las moléculas extracelulares como la capacidad de crecimiento intrínseca de la neurona influyen en el éxito regenerativo. ${ }^{2}$ Algunas lesiones de la médula espinal y de los nervios periféricos alteran la calidad de vida de los pacientes que las padecen. ${ }^{3,4}$

El desarrollo de técnicas de regeneración de tejidos del sistema nervioso tiene como objetivo proveer métodos para reparación funcional y restauración de la función motora y sensorial. ${ }^{6,7}$ Para regenerar nervios periféricos se ha probado el trasplante de las células de Schwann como una alternativa, quienes sintetizan $y$ secretan factores neurotróficos, moléculas de adhesión L1 y matriz extracelular para guiar y promover el crecimiento axonal y mielinización. ${ }^{5,8,9}$ También se ha demostrado que la aplicación de terapias moleculares combinada con terapias celulares y andamios o scaffolds (en inglés) de biomateriales en el sistema nervioso central ha incrementado el entendimiento de los requerimientos y condiciones adecuadas para la reparación de la médula espinal. ${ }^{10}$

Además, se ha usado el trasplante de células madre (incluso las inducidas pluripotentes), basándose en su habilidad de autorrenovación y proliferación, así como su plasticidad. ${ }^{11,12}$ Aunque el uso de células madre puede contribuir a mantener la homeostasis celular y acelerar la cicatrización también se han asociado a la génesis de tumores, la metástasis y otras enfermedades. ${ }^{13}$

El uso de terapias de medicina regenerativa que promuevan la regeneración nerviosa se han convertido en estrategias prometedoras.
Estos procedimientos se basan en el uso de células madre, diferentes tipos de andamios, materiales y moléculas bioactivas que serán abordados en esta revisión.

\section{Soportes usados en regeneración nerviosa}

Las lesiones de nervios periféricos son un problema clínico global, por su significativo efecto sobre la calidad de vida de los pacientes, por esto, han surgido estrategias encaminadas a reparar dichas lesiones y entre estas están los injertos, que a su vez han presentado una evolución tanto de los materiales utilizados como de su estructura. Se utilizaron en principio los injertos compuestos de materiales naturales provenientes de tejidos autólogos, alogénicos y xenogénicos, después como alternativa se utilizaron materiales como polímeros derivados de componentes de la matriz extracelular, polisacáridos y proteínas; con respecto a la estructura, al inicio se construían estructuras bidimensionales y actualmente se han elaborado compuestos de estructura tridimensional (3D). Existen estudios en los que han utilizado autoinjertos de nervios para reparar grandes defectos de nervios periféricos, sin embargo, debido a las fuentes limitadas de autoinjertos y a la inevitable pérdida sensorial del área donadora después de obtener injerto nervioso, se hace difícil satisfacer el creciente requerimiento de reparar daños nerviosos periféricos. ${ }^{14,15}$

Muchos investigadores han intentado desarrollar biomateriales más efectivos para crear alternativas a los injertos y promover la regeneración nerviosa, las estructuras que recrean el microambiente en 3D para ayudar al crecimiento y regeneración del tejido reciben el nombre de andamios, soportes o scaffolds, que deben cumplir con características como flexibilidad, biocompatibilidad y resistencia al colapso estructural durante la implantación. .,16,17 Guan S y colaboradores usaron tejido neural embrionario y diseccionaron muestras 
del hipocampo de ratas Sprague-Dawley, fabricaron mediante liofilización andamios porosos de quitosano $(\mathrm{Cs}) /$ gelatina $(\mathrm{Gel})$ que contenían ácido hialurónico (HA) y sulfato de heparán (HS), posteriormente los ensayos de viabilidad celular, microscopía electrónica de barrido y microscopía de fluorescencia revelaron que la presencia de HA y HS en los andamios promovió significativamente la adhesión de las células progenitoras y del tallo neural (NS/PC) y apoyó el crecimiento a largo plazo en el entorno tridimensional $\mathrm{y}$ diferenciación mutilinaje con una diferenciación neuronal. Concluyendo que los andamiajes compuestos de $\mathrm{Cs} / \mathrm{Gel} /$ HA/HS eran adecuados para la adhesión, supervivencia y crecimiento de las células neurales y podrían ofrecer nuevas opciones para aplicaciones de ingeniería de tejidos neurales. ${ }^{18}$

S. H. I Qin y colaboradores estudiaron andamios compuestos por colágeno y el factor de crecimiento fibroblástico (FGF), para regeneración en un modelo de hemisección de médula espinal, los resultados mostraron que esta estrategia mejora las tasas de sobrevida y las capacidades motoras de las ratas a las que se les realizó el implante con este andamio, lo que sugiere que podría ser una herramienta empleada para favorecer la recuperación de la funcionalidad perdida. ${ }^{19}$

Otros scaffolds o andamios disponibles para regeneración neural a escala submicrómica con conductividad eléctrica y actividad neurotrófica, fueron elaborados con nanofibras electrohiladas de poli ácido láctico-co-ácido glicólico (PLGA) combinadas con factor de crecimiento nervioso (NGF) que se inmovilizó químicamente sobre la superficie de las fibras. Estas fibras soportaron la formación de neuronas y su crecimiento, una estrategia prometedora en la conducción nerviosa. ${ }^{20}$

\section{Regeneración de neuronas}

Las enfermedades neurodegenerativas se caracterizan por la pérdida temprana de la función y posterior muerte de las neuronas, además de la falta de regeneración celular, afectando subpoblaciones neuronales específicas que muestran una susceptibilidad única al desarrollo de este tipo de enfermedades. ${ }^{21} \mathrm{La}$ esclerosis lateral amiotrófica representa un ejemplo de estas alteraciones, donde ocurre una degeneración de las neuronas motoras que lleva a debilidad muscular, parálisis y una muerte temprana del paciente. ${ }^{22}$ Estudios recientes han proporcionado información sobre regeneración neuronal, un proceso altamente regulado y complejo. Sin embargo, los mecanismos moleculares que determinan la capacidad regenerativa de las células madre y la capacidad de las neuronas recién generadas para dirigir sus axones hacia objetivos específicos siguen siendo esquivos. ${ }^{23}$

En este sentido, algunos investigadores han empleado neurotransmisores como la dopamina y la serotonina para controlar la neurogénesis de las lesiones. Scott y colaboradores en el año 2015 utilizaron neurotransmisores para apoyar el proceso de regeneración neuronal, demostrando que la serotonina promueve la regeneración de neuronas motoras adultas, además de incrementar la proliferación de células progenitoras de neuronas motoras embrionarias y células adultas como las células gliales epéndimo radiales. ${ }^{24}$

El desarrollo coordinado del tronco encefálico y neuronas diana espinales es fundamental para la generación de un sistema locomotor que funcione con precisión. Las señales que coinciden con el desarrollo de estas regiones alejadas del sistema nervioso central se pueden redistribuir durante la regeneración de la médula espinal. Reimer y colaboradores explican que los axones descendentes tienen una gran influencia en la plasticidad de las células progenitoras de la columna vertebral durante el desarrollo 
y después de una lesión en los vertebrados; estos investigadores demostraron que las proyecciones dopaminérgicas descendentes del cerebro promueven la generación de neuronas motoras a expensas de las interneuronas V2 en la médula espinal de pez cebra en desarrollo mediante la activación del receptor $\mathrm{D} 4 \mathrm{a}$, que actúa en la vía del erizo. La inhibición de esta señal esencial durante la neurogénesis temprana conduce a una reducción duradera del número de neuronas motoras y al deterioro de las respuestas motoras. Es importante destacar que durante la regeneración exitosa de la médula espinal en el pez cebra adulto, la dopamina endógena promueve la generación de neuronas motoras espinales, y los agonistas de la dopamina aumentan este proceso, describiendo un mecanismo de control supraespinal para el desarrollo y la regeneración de tipos específicos de células espinales que utilizan dopamina como señal. 25

Teniendo en cuenta que las neurotrofinas desempeñan papeles críticos en el desarrollo del sistema nervioso y en la plasticidad sináptica en el adulto, protegiendo a las neuronas de degeneración, mejorando la diferenciación de las células madre neurales mediante la activación de receptores de tirosina quinasa (trk) y de otras vías de señalización. Song y colaboradores, propusieron que el factor neurotrófico derivado del cerebro (BDNF) aplicado periféricamente puede actuar en la regeneración de los axones centrales de las neuronas sensoriales ascendentes. Para esto realizaron de una lesión de acondicionamiento del nervio ciático como modelo para aumentar la expresión de BDNF endógeno en las neuronas sensoriales e inyectaron BDNF exógeno en el nervio periférico o en los tejidos. El suministro de BDNF exógeno en el nervio ciático o en la almohadilla de la pata de ratas aumentó significativamente el número de neuronas de ganglios de la raíz dorsal DRG (del inglés Dorsal Root Ganglia) y regeneró axones sensoriales en la médula espinal lesionada. Los autores concluyeron que, BDNF endógeno en DRG y la médula espinal son necesarios para la regeneración mejorada de las neuronas sensoriales ascendentes después de la lesión del nervio ciático y el BDNF aplicado periféricamente puede tener efectos terapéuticos en la lesión de la médula espinal. ${ }^{26}$

De igual forma se han realizado estudios para otro tipo de lesiones del sistema nervioso central ocasionadas por proyectiles, como la lesión penetrante cerebral, este tipo de lesiones causan muerte celular extensiva y pérdida permanente del parénquima cerebral. En un estudio publicado en el año 2015, los investigadores elaboraron un andamio para la reparación de este tipo de lesión, teniendo en cuenta que la lesión penetrante cerebral y otras lesiones cerebrales causadas por traumas pueden presentarse factores que inhiben el crecimiento, así como también inhiben la regeneración del tejido lesionado. Los autores decidieron utilizar el receptor soluble Nogo $(\mathrm{sNgR})$ para prevenir la acción de las proteínas de mielina en la superficie de la lesión y permitir la regeneración. Los soportes utilizados, estaban compuestos por colágeno tipo I y liberaron el agente terapéutico $\mathrm{sNgR}$ luego de ser implantados en una lesión penetrante cerebral en 7 ratas, para comprobar el rendimiento de estos scaffolds, a otro grupo de 7 ratas se les implantaron andamios que no contenían el agente. Los resultados obtenidos mostraron que el grupo de animales implantados con el andamio con $\mathrm{sNgR}$ cubrían de mejor forma la superficie lesionada, favorecieron su vascularización, sin infiltración de otras células como macrófagos. ${ }^{27}$

Regeneración de axones.

A través de mecanismos complejos que guían a los axones hacia las rutas apropiadas, el crecimiento axonal conduce a la formación 
funcionamiento óptimo y del sistema neuronal. El daño a estas redes puede ser reparado por procesos neuro regenerativos que a su vez pueden restablecer las sinapsis entre los axones lesionados y los terminales postsinápticos. La orientación axonal y la respuesta neuro regenerativa dependen del apropiado crecimiento axonal, de las respuestas correctas del cono de crecimiento axonal a las moléculas señalizadoras (principalmente proteínas de fusión de membrana del complejo SNARE, receptores de proteínas de fijación soluble de NSF), así como las sinapsis correctas con objetivos apropiados..$^{28}$

En la regeneración de axones se ha utilizado terapia génica para mejorar los parámetros estructurales y funcionales de células y tejido nervioso después de una lesión en la médula espinal, como se demuestra en estudio realizado en la Universidad Estatal de Medicina de Kazan en Rusia, donde investigaron el potencial de las células mononucleares de la sangre del cordón umbilical (UCB$\mathrm{MCs}$ ), genéticamente modificadas con factor de crecimiento endotelial vascular (VEGF) y factor neurotrófico derivado de células gliales (GNDF), para esto utilizaron un vector adenoviral con el objetivo de liberar estos factores de crecimiento. En el estudio se examinó la eficacia en tejido faltante, cicatriz glial severa, extensión de la regeneración axonal y recuperación de la función motora. Los resultados mostraron que el vehículo adenoviral fue efectivo y estable para las células neuronales in vivo. ${ }^{29}$ Este estudio al igual que otros estudios demostraron que el trasplante de células genéticamente modificadas tiene un efecto estimulador en la regeneración del sistema nervioso central después de un trauma o lesión. . $^{30,31}$

Después de una lesión de nervios periféricos, los axones desgarrados pueden regenerarse y reinervar órganos importantes, sin embargo, la reinervación de órganos distales y recuperación funcional son por lo general deficientes debido a que la regeneración de los axones es aleatoria lo cual resulta en una reinervación irregular. ${ }^{32}$ Ante este tipo de lesiones, se ha mostrado que los factores neurotróficos (NTFs) están involucrados en las vías del crecimiento de los axones. $^{33}$ Un estudio realizado por el Instituto de Neurociencias y el Departamento de Biología Celular de la Universidad Autónoma de Barcelona en España, tuvo en cuenta los niveles de los NTFs y sus concentraciones para analizar el efecto de esos factores de crecimiento sobre la regeneración de axones motores y sensoriales utilizando modelos in vitro e in vivo, debido a que altos niveles de NTFs o su liberación durante un tiempo prolongado podría inducir retroceso en términos de la regeneración, en el estudio se aplicaron dosis óptimas para estimular la regeneración axonal motora y sensorial para diferentes NTFs como GDNF (glial cell line-derived NTF), FGF-2, factor de crecimiento nervioso (NGF), NT-3 (neurotrofina 3) y BDNF (brain-derived neurotrophic factor). Observaron que la aplicación de GDNF y FGF-2 proporcionaron la mayor regeneración motora y neuro sensorial, la aplicación de NGF y NT-3 mejoró selectivamente el crecimiento neurítico sensorial in vitro que se pierde en el modelo in vivo, y que la aplicación de BDNF en dosis seleccionadas promueve el crecimiento axonal motor in vivo e in vitro. ${ }^{34}$

Aunque existen factores limitantes en el uso de estas estrategias, muchos de los resultados son prometedores en la mejora tisular, fisiológica y funcional en lesiones que implican daño de los axones.

\section{Regeneración de nervios}

La reparación o regeneración de nervios es de vital importancia ya que este tipo de lesiones, principalmente relacionadas 
con traumatismos, tumores y lesiones iatrogénicas conllevan a déficits neurológicos y discapacidad funcional, en la búsqueda de reparar este tejido se han implementado diferentes técnicas, que van desde la implantación de injertos, pasando por terapia celular y molecular, hasta la implantación de andamios 3D. Con respecto a las células madre las que sehan usado con mas frecuencia en regeneración de nervios periféricos, son las células madre embrionarias (ESCs), células madre neuronales (NSCs), células madre mesenquimales (MSCs), células madre derivadas de la médula ósea (BMSCs) y derivadas de tejido adiposo (ADSCs), las células madre derivadas del líquido amniótico (ATDSCs) y derivadas del cordón umbilical (UC-MSCs), las células madre precursoras derivadas de la piel (SKP-SCs) y las células derivadas de folículos pilosos (HFSCs). Ciertas células madre contribuyen con el mejoramiento de la acción neurotrófica al proporcionar un microambiente benéfico para las células neuronales, un ejemplo son las MSCs que sintetizan y liberan una variedad de factores de crecimiento neurotróficos, las SKP-SCs y las ADSCs incrementan y regulan, respectivamente, la expresión de estos factores. ${ }^{35}$

En un estudio realizado por Al-zer y colaboradores usaron células de Schwann (SC) para la regeneración del nervio periférico. La pulpa dental de los humanos adultos contiene diferentes poblaciones de células madre, que muestran una amplia diversidad y potenciales, las células de Schwann derivadas de células madre de la pulpa dental (DPSC) mostraron crecimiento adecuado en cultivo, y la inducción de la diferenciación en SC. La población de DPSC se podrá tener en cuenta en el futuro para la regeneración de nervios periféricos después de su inducción en $\mathrm{SC}$ in vitro, como una fuente alternativa superior de SC en comparación con la fuente de células autóloga o de donantes de nervios. Las DPSC son, rentables y con tasas de proliferación aceptables y no requieren procedimientos quirúrgicos complicados. ${ }^{36}$

Otro estudio realizado por Sowa y colaboradores en el año 2017, generaron células de Schwann funcionales mediante procedimientos de reprogramación de células somáticas, demostrando su capacidad para promover la regeneración de nervios periféricos. Fibroblastos humanos normales se convirtieron fenotípicamente en $\mathrm{SC}$ mediante la transducción de los genes SOX10 y Krox20, seguido de un cultivo por 10 días, lo que resultó en aproximadamente el $43 \%$ de células de Schwann convertidas directamente (dSC). Finalmente, las células modificadas genéticamente se sembraron en un andamio de hidrogel que posteriormente se implantó en una lesión del nervio ciático de ratas. Las dSC expresaron proteínas específicas de SC y secreción de factores neurotróficos. Las dSC también mostraron capacidad de formación de mielina tanto in vitro como in vivo. Además, el trasplante de las dSC en el nervio ciático en ratones resultó en una regeneración del nervio significativamente acelerada y en una función motora mejorada a un nivel comparable al del trasplante de los $\mathrm{SC}$ obtenidos de un nervio periférico. Los autores concluyen que las dSC inducidas con este protocolo podrían ser una nueva alternativa terapéutica en regeneración no solo de nervios periféricos, sino también de nervios centrales, así como para trastornos neurodegenerativos relacionados con la disfunción de las SC, por lo tanto con aplicaciones clínicas no solo para lesiones nerviosas periféricas sino también para lesiones cerebrales y de la médula espinal y para trastornos desmielinizados del SNC, incluida la esclerosis múltiple. ${ }^{37}$

Yurie $\mathrm{H}$ y colaboradores estudiaron la eficacia de bioandamios en 3D en la regeneración del modelo del nervio ciático en ratones, como una búsqueda de nuevas herramientas para el tratamiento de lesiones 
de los nervios periféricos. En este trabajo se desarrollaron 6 andamios a partir de fibroblastos dérmicos humanos utilizando una bioimpresora 3D. Se usaron 12 ratas macho adultas que tenían una transección del nervio ciático derecho, en seis ratas se utilizaron los bio andamios 3D para puentear una separación de nervio de $5 \mathrm{~mm}$ con andamios de $8 \mathrm{~mm}$, en las otras 6 ratas se utilizaron tubos de silicona para comparar los efectos entre uno y otro andamio. Los resultados del análisis cinemático revelaron que el ángulo del dedo del pie al hueso metatarsiano era significativamente más alto cuando se utilizaron los bioandamios 3D que los andamios de silicona, los estudios electrofisiológicos revelaron un potencial de acción muscular fue significativamente mayor con el bioandamio 3D que con el andamio de silicona, por último los estudios histológicos y morfométricos mostraron presencia de células neurales en todas las regiones de los nervios regenerados y la presencia de muchos axones bien mielinizados cuando se utilizaron los bioandamios 3D. ${ }^{38}$

Para la reparación de nervios periféricos se ha descrito el método de Sondell, que utiliza andamios de nervios descelularizados usando tejido del nervio ciático, este método ha demostrado eficiencia ya que remueve células nerviosas y mielina en el sitio de la lesión del nervio y reduce la respuesta inmune después de los xenoinjertos de nervio. ${ }^{39}$ Hudson propuso un método que es una mejora al método expuesto anteriormente, donde el andamio diseñado por su grupo de investigación mantiene la membrana y los componentes estructurales, mejorando la regeneración del nervio después del trasplante..$^{40}$

En un estudio realizado en el departamento de neurocirugía y la Universidad de Sun Yat-sen en Guangzhou, China el método de Hudson fue mejorado removiendo completamente los componentes de mielina y preparando scaffolds acelulares desmielinizados para tejido nervioso. Los soportes fueron fabricados a partir del nervio ciático de ratas y mostraron que estos scaffolds a diferencia de los utilizados en el método Hudson permiten regenerar nervios periféricos libres de mielina, mientras que en el método Hudson aunque se daba la regeneración del nervio periférico persisten componentes de mielina, adicionalmente, los nervios regenerados a partir del scaffold desmielinizado eran más gruesos y densos que los regenerados a partir del scaffold propuesto por Hudson. ${ }^{40}$

\section{Conclusiones}

La ingeniería de tejidos nos brinda ideas interesantes sobre el diseño de andamios con propiedades neuroconductoras, neuroinductoras, mecánicas, de porosidad, biológicas y compatibilidad. Estos andamios también pueden evitar infecciones, múltiples cirugías y costos adicionales para el paciente. El mayor desafío con los andamios reside en la elección de biomateriales con la combinación correcta de propiedades.

Cada tipo de lesión requerirá un sistema de administración optimizado y específico, con diferentes combinaciones de células y biomoléculas. Del tipo de células utilizadas y la difusión de sustancias bioactivas dependerá en gran medida la regeneración tisular.

Las terapias basadas en células madre, andamios, factores neurotróficos y moléculas bioactivas, no invasivas en combinación con rehabilitación, allanarán el camino del futuro de la medicina regenerativa neuronal. 


\section{Resumen}

Introducción: Este artículo presenta avances de la medicina regenerativa y la ingeniería de tejidos orientados a la regeneración de neuronas, de axones y nervios. Revisamos las técnicas que existen actualmente, las más utilizas o prometedoras, en la búsqueda de avances para regenerar este tipo de tejidos. Objetivo: Con esta revisión queremos describir el conocimiento actual sobre la medicina regenerativa y la ingeniería de tejidos orientados a la reparación de tejidos nerviosos. Metodología: Para desarrollar esta revisión se realizó una búsqueda de artículos entre los años 2007 y el 2018, la búsqueda se restringió a los artículos que incluyeran dentro de sus palabras clave; Ingeniería tisular, Enfermedades Neurodegenerativas, Medicina regenerativa, Regeneración axonal, Regeneración neuronal, Regeneración tisular. Con el fin de seleccionar los artículos más adecuados, se realizó una búsqueda exhaustiva en bases de datos como Springer, Medline Ebsco y Science direct. Conclusiones: Se mencionan técnicas como implantación de injertos, terapia celular y terapia molecular e implantación de andamios $3 D$ para regeneración de neuronas, axones y nervios; a partir de esta revisión pudimos observar que estas técnicas en su mayoría funcionan mejor cuando se combinan, aprovechando las ventajas de cada una para promover la regeneración de los diferentes tejidos nerviosos.

Palabras clave: Ingeniería tisular, Enfermedades Neurodegenerativas, Medicina regenerativa.

\section{Referencias Bibliográficas}

1. Hutcheson JA, Kimberley MO. A pragmatic approach to characterising insect communities in New Zealand: Malaise trapped beetles. N Z J Ecol. 1999;23(1):69-79. doi:10.1016/ S1474-4422(17)30299-5

2. Addendum A, Aga O, Ahrens MB, et al. NIH Public Access. J Neurosci. 2012;7(1):n/a-n/a. doi:10.1007/400

3. Martín-López E, Alonso FR, Nieto-Díaz M, Nieto-Sampedro M. Chitosan, gelatin and poly(L-Lysine) polyelectrolyte-based scaffolds and films for neural tissue engineering. J Biomater Sci Polym Ed. 2012;23(1-4):207232. doi:10.1163/092050610X546426

4. Vacanti JP1 LR. Tissue engineering: the design and fabrication of living replacement devices for surgical recons- truction and transplantation. Lancet. 1999;354(1):132-134.

5. Yao L, Daly W, Newland B, et al. Improved axonal regeneration of transected spinal cord mediated by multichannel collagen conduits functionalized with neurotrophin-3 gene. Gene Ther. 2013;20(12):1149-1157. doi:10.1038/ gt.2013.42

6. Seil JT, Webster TJ. Electrically active nanomaterials as improved neural tissue regeneration scaffolds. Wiley Interdiscip Rev Nanomedicine Nanobiotechnology. 2010;2(6):635-647. doi:10.1002/wnan.109

7. An Y, Tsang KKS, Zhang H. Potential of stem cell based therapy and tissue engineering in the regeneration of the central nervous system. Biomed Mater. 2006;1(2). doi:10.1088/1748$6041 / 1 / 2 / R 02$ 
8. Pan M, Wang X, Chen Y, et al. Tissue engineering with peripheral blood-derived mesenchymal stem cells promotes the regeneration of injured peripheral nerves. Exp Neurol. 2017;292:92-101. doi:10.1016/j.expneurol.2017.03.005

9. Subramanian A, Krishnan U, Sethuraman S. Development of biomaterial scaffold for nerve tissue engineering: Biomaterial mediated neural regeneration. J Biomed Sci. 2009;16(1):108. doi:10.1186/1423-0127-16-108

10. Kuhlengel KR, Bunge MB, Bunge RP. Implantation of cultured sensory neurons and schwann cells into lesioned neonatal rat spinal cord. I. Methods for preparing implants from dissociated cells. J Comp Neurol. 1990;293(1):6373. doi:10.1002/cne.902930106

11. Willerth SM. Neural tissue engineering using embryonic and induced pluripotent stem cells. Stem Cell Res Ther. 2011;2(2):1-9. doi:10.1186/ scrt58

12. Montgomery A, Wong A, Gabers N, Willerth SM. Engineering personalized neural tissue by combining induced pluripotent stem cells with fibrin scaffolds. Biomater Sci. 2015;3(2):401413. doi:10.1039/c4bm00299g

13. Hansmann F, Pringproa K, Ulrich R, et al. Highly Malignant Behavior of a Murine Oligodendrocyte Precursor Cell Line Following Transplantation into the Demyelinated and Nondemyelinated Central Nervous System. Cell Transplant. 2012;21(6):1161-1175. doi:10.3727/096368911X627444

14. James Noble; Catherine A. Munro; Vannemreddy S. S. V. Prasad; Rajiv Midha. Analysis of Upper and Lower Extremity Peripheral Nerve Injuries in a Population of Patients with Multiple Injuries. J Trauma Inj Infect Crit Care. 1998;45(1)(116-122).
15. Gu X, Ding F, Williams DF. Neural tissue engineering options for peripheral nerve regeneration. Biomaterials. 2014;35(24):6143$6156 . \quad$ doi:10.1016/j.biomaterials.2014.04.064

16. Hu J, Tian L, Prabhakaran MP, Ding X, Ramakrishna S. Fabrication of nerve growth factor encapsulated aligned poly( $\varepsilon$-caprolactone) nanofibers and their assessment as a potential neural tissue engineering scaffold. Polymers (Basel). 2016;8(2). doi:10.3390/ polym8020054

17. GhoshMitra S, Diercks DR, Mills NC, Hynds DAL, Ghosh S. Role of engineered nanocarriers for axon regeneration and guidance: Current status and future trends. Adv Drug Deliv Rev. 2012;64(1):110-125. doi:10.1016/j. addr.2011.12.013

18. Guan S, Zhang XL, Lin XM, Liu TQ, Ma XH, Cui ZF. Chitosan/gelatin porous scaffolds containing hyaluronic acid and heparan sulfate for neural tissue engineering. J Biomater Sci Polym Ed. 2013;24(8):999-1014. doi:10.108 0/09205063.2012.731374

19. Shi Q, Gao W, Han XL, et al. Collagen scaffolds modified with collagen-binding bFGF promotes the neural regeneration in a rat hemisected spinal cord injury model. Sci China Life Sci. 2014;57(2):232-240. doi:10.1007/ s11427-014-4612-7

20. Lee JY, Bashur CA, Milroy CA, Forciniti L, Goldstein AS, Schmidt CE. Nerve growth factor-immobilized electrically conducting fibrous scaffolds for potential use in neural engineering applications. IEEE Trans Nanobioscience. 2012;11(1):15-21. doi:10.1109/TNB.2011.2159621

21. Dametti S, Faravelli I, Ruggieri M, Ramirez A, Nizzardo M, Corti S. Experimental Advances Towards Neural Re- 
generation from Induced Stem Cells to Direct In Vivo Reprogramming. Mol Neurobiol. 2016;53(4):2124-2131. doi:10.1007/s12035-015-9181-7

22. Rizzo F, Riboldi G, Salani S, et al. Cellular therapy to target neuroinflammation in amyotrophic lateral sclerosis. Cell Mol Life Sci. 2014;71(6):999-1015. doi:10.1007/ s00018-013-1480-4

23. Yu CR, Wu Y. Regeneration and rewiring of rodent olfactory sensory neurons. Exp Neurol. 2017;287:395-408. doi:10.1016/j.expneurol.2016.06.001

24. Barreiro-Iglesias A, Mysiak KS, Scott AL, et al. Serotonin Promotes Development and Regeneration of Spinal Motor Neurons in Zebrafish. Cell Rep. 2015;13(5):924-932. doi:10.1016/j. celrep.2015.09.050

25. Reimer MM, Norris A, Ohnmacht J, et al. Dopamine from the Brain Promotes Spinal Motor Neuron Generation during Development and Adult Regeneration. Dev Cell. 2013;25(5):478-491. doi:10.1016/j.devcel.2013.04.012

26. Song XY, Li F, Zhang FH, Zhong JH, Zhou XF. Peripherally-derived BDNF promotes regeneration of ascending sensory neurons after spina cord injury. PLoS One. 2008;3(3). doi:10.1371/journal.pone.0001707

27. Elias PZ SM. Treatment of penetrating brain injury in a rat model using collagen scaffolds incorporating soluble Nogo receptor. J Tissue Eng Regen Med. 2015;9((2)):137-150.

28. Ulloa F, Cotrufo T, Ricolo D, Soriano E, Araújo SJ. SNARE complex in axonal guidance and neuroregeneration. Neural Regen Res. 2018;13(3):386392. doi:10.4103/1673-374.228710

29. Mukhamedshina YO, Garanina EE, Masgutova GA, et al. Assessment of glial scar, tissue sparing, behavioral recovery and axonal regeneration following acute transplantation of genetically modified human umbilical cord blood cells in a rat model of spinal cord contusion. PLoS One. 2016;11(3):1-16. doi:10.1371/journal. pone. 0151745

30. Yan HB1, Zhang ZM, Jin DD, Wang XJ LK. The repair of acute spinal cord injury in rats by olfactory ensheathing cells graft modified by glia cell line-derived neurotrophic factor gene in combination with the injection of monoclonal antibody IN-1. Chinese J Surg. 2009;47(23):1817-1820.

31. Kim HM, Hwang DH, Lee JE, Kim SU, Kim BG. Ex Vivo VEGF delivery by neural stem cells enhances proliferation of glial progenitors, angiogenesis, and tissue sparing after spinal cord injury. PLoS One. 2009;4(3):1-10. doi:10.1371/journal.pone.0004987

32. Valero-Cabré A, Navarro X. Functional impact of axonal misdirection after peripheral nerve injuries followed by graft or tube repair. J Neurotrauma. 2002;19(11):1475-1485. doi:10.1089/089771502320914705

33. Markus A, Patel TD, Snider WD. Neurotrophic factors and axonal growth. Curr Opin Neurobiol. 2002;12(5):523-531. doi:10.1016/ S0959-4388(02)00372-0

34. Santos D, Gonzalez-Perez F, Navarro X, Del Valle J. Dose-Dependent Differential Effect of Neurotrophic Factors on in Vitro and in Vivo Regeneration of Motor and Sensory Neurons. Neural Plast. 2016;2016. doi:10.1155/2016/4969523

35. Jiang L, Jones S, Jia X. Stem cell transplantation for peripheral nerve regeneration: Current options and opportunities. Int J Mol Sci. 2017;18(1):1-17. doi:10.3390/ijms 18010094

36. Al-Zer H, Kalbouneh H. Dental pulp stem cells-derived schwann cells for 
peripheral nerve injury regeneration. Neural Regen Res. 2015;10(12):19451946. doi:10.4103/1673-5374.172309.

37. For A. P ROTOCOLS AND M ANUFACTURING FOR C ELL -B ASED T HERAPIES Direct Conversion of Human Fibroblasts into Schwann Cells that Facilitate Regeneration of Injured Peripheral Nerve In Vivo. 2017:12071216.

38. Yurie H, Ikeguchi R, Aoyama T, et al. The efficacy of a scaffold-free bio 3D conduit developed from human fibroblasts on peripheral nerve regeneration in a rat sciatic nerve model. PLoS One. 2017;12(2):1-16. doi:10.1371/journal. pone. 0171448
39. Sondell M, Lundborg G, Kanje M. Regeneration of the rat sciatic nerve into allografts made acellular through chemical extraction. Brain Res. 1998;795(1-2):44-54. doi:10.1016/ S0006-8993(98)00251-0

40. Cai M, Huang T, Hou B, Guo Y. Role of Demyelination Efficiency within Acellular Nerve Scaffolds during Nerve Regeneration across Peripheral Defects. Biomed Res Int. 2017;2017. doi:10.1155/2017/4606387

Correspondencia:

Lina Andrea Gómez

linagore@unisabana.edu.co

(55) 8615555 - (55) 3113303448 